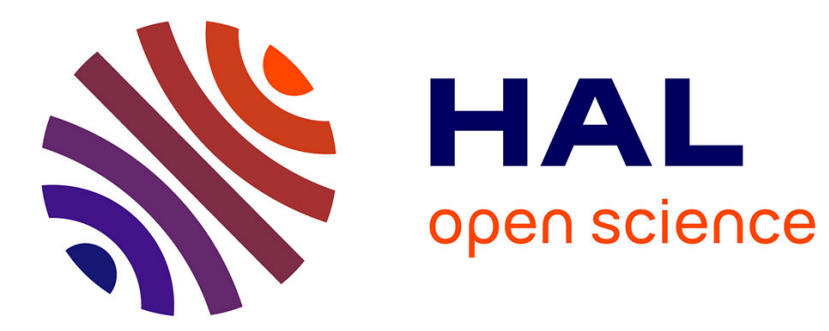

\title{
Hybrid Filter Bank A/D conversion systems applied to future telecommunication scenarios
}

Caroline Lelandais-Perrault, Zhiguo Song, Daniel Poulton

\section{To cite this version:}

Caroline Lelandais-Perrault, Zhiguo Song, Daniel Poulton. Hybrid Filter Bank A/D conversion systems applied to future telecommunication scenarios. IEEE International NEWCAS Conference (NEWCAS-TAISA'09), Jun 2009, Toulouse, France. 4 p. hal-00444399

\section{HAL Id: hal-00444399 \\ https://hal-centralesupelec.archives-ouvertes.fr/hal-00444399}

Submitted on 6 Jan 2010

HAL is a multi-disciplinary open access archive for the deposit and dissemination of scientific research documents, whether they are published or not. The documents may come from teaching and research institutions in France or abroad, or from public or private research centers.
L'archive ouverte pluridisciplinaire HAL, est destinée au dépôt et à la diffusion de documents scientifiques de niveau recherche, publiés ou non, émanant des établissements d'enseignement et de recherche français ou étrangers, des laboratoires publics ou privés. 


\title{
Hybrid Filter Bank A/D conversion systems applied to future telecommunication scenarios
}

\author{
Caroline Lelandais-Perrault, Zhiguo Song and Daniel Poulton \\ SUPELEC, Gif-sur-Yvette, FRANCE \\ Email: firstname.lastname@supelec.fr
}

\begin{abstract}
Hybrid Filter Banks (HFB) A/D converters (ADC) are attractive to software-defined radio applications. Starting from a given sampling rate, they enlarge the conversion bandwidth. Also, it is possible to adapt the conversion characteristics (e.g. bandwidth and resolution) by software control. HFB have been studied in the context of the ANR VersaNum project. This work proposes optimal HFBs and a calibration technique to compensate the mismatch between the analog part and the digital part. Results are given for future telecommunication scenarios.
\end{abstract}

\section{INTRODUCTION}

VersaNum is a 3-year multi-partner project which has been approved by the RNRT (Réseau National de Recherche en Télécommunications) in 2006 and financed by the ANR (Agence Nationale de la Recherche). This project addresses the analog-to-digital conversion needs for the future communications systems. Several scenarios have been defined for the future A/D conversion needs. Two of them have been selected here because they deal with main steps in the evolution of future communication systems. The first scenario is a "multistandard" scenario. It defines a system that could alternatively treat one of the following standards: GSM, UMTS, WiFi or WiMax. The specifications of the A/D converters are given in Table I. The second scenario is called "sensing in the TV band". The system should be able to alternatively sense a very wide band [470-960] $\mathrm{MHz}$ (including the TV band and the GSM band) with a rather low resolution and communicate on a channel band of $200 \mathrm{kHz}$ with a high resolution. The A/D conversion requirements for each mode are given in Table II.

TABLE I

SCENARIO 1 - A/D CONVERSION REQUIREMENTS

\begin{tabular}{|l||c|c|}
\hline Standard & Channel bandwidth & Dynamic range \\
\hline \hline WiMax & $25 \mathrm{MHz}$ & $44 \mathrm{~dB}$ \\
\hline WiFi & $22 \mathrm{MHz}$ & $52 \mathrm{~dB}$ \\
\hline UMTS & $5 \mathrm{MHz}$ & $81 \mathrm{~dB}$ \\
\hline GSM & $200 \mathrm{kHz}$ & $82 \mathrm{~dB}$ \\
\hline
\end{tabular}

TABLE II

SCENARIO 2 - A/D CONVERSION REQUIREMENTS

\begin{tabular}{|l||c|c|}
\hline Mode & Bandwidth & Dynamic range \\
\hline \hline Sensing & $490 \mathrm{MHz}$ & $36 \mathrm{~dB}$ \\
\hline Communication & $200 \mathrm{kHz}$ & $82 \mathrm{~dB}$ \\
\hline
\end{tabular}

In order to achieve these requirements and to overcome technological limitations, the A/D conversion systems should be versatile, i.e. it should be possible to adapt the conversion characteristics (resolution and bandwidth) by software. We call this "frequency focusing".

HFB ADC is a good candidate. The HFBs are multi-rate systems that use a 3-stage process: frequency band decomposition through an analog analysis filter bank; A/D conversion; and finally, reconstruction through a digital synthesis filter bank. HFBs are interesting because it enlarges the conversion bandwidth for a given sampling rate on each channel. Moreover, the A/D conversion characteristics may be changed by reprogramming the digital part.

In VersaNum, HFBs have been optimized for typical scenarios. Also, the major drawback of HFB which is the very high sensitivity to the mismatch between the analog and the digital parts have been studied. Calibration techniques are proposed. An HFB prototype has been designed to validate the proposed architecture. At the end of the project, the developed methods will be tested on the prototype.

This paper presents the main results concerning HFB obtained during the project until now. Section II describes the HFB and their optimization for above scenarios. Section III addresses the versatility of HFB in terms of "frequency focusing". Section IV presents the calibration solutions. Section $\mathrm{V}$ presents the specifications of the HFB prototype. In each section, simulation results are given.

\section{HFB PRESENTATION}

\section{A. Review of HFB theory}

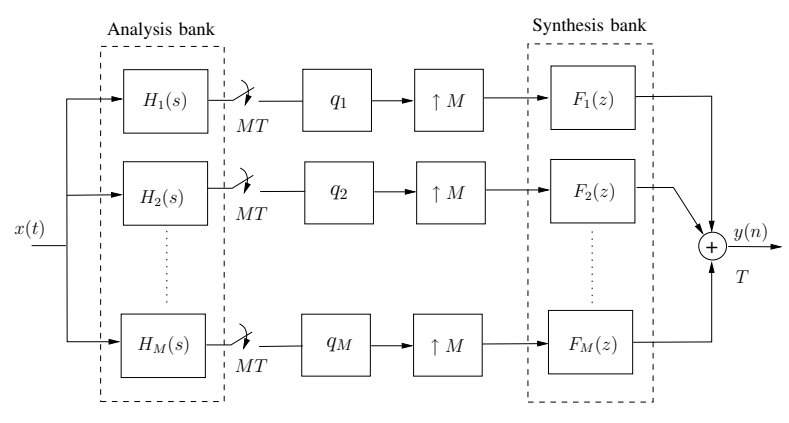

Fig. 1. Maximally decimated hybrid filter bank structure

Figure 1 shows a $M$-channel maximally decimated HFB. $x(t)$ is the real input signal to be digitized at $1 / T$ rate and $y(n)$ is the digital output of the filter bank. $H_{m}(s)$ are the 
transfer functions of the continuous-time analysis filter bank and $F_{m}(z)$ are the transfer functions of the discrete-time synthesis filter bank, with $m \in\{1,2, \ldots, M\}$. The blocks $q_{1}, \ldots, q_{M}$ are the branch quantizers. After analog filtering, the branch signals $x_{m}(t)$ are sampled at a rate of $1 / M T$. It is assumed that the input signal is strictly bandlimited to $B$. In this case, the Nyquist criterion for sampling with an effective sampling frequency of $1 / T=2 B$ without aliasing can be fulfilled. Ignoring the quantizers, the Fourier transform of the HFB output signal $y(n)$ is [7]:

$$
Y\left(e^{\mathrm{j} \omega}\right)=\frac{1}{T} \sum_{p=-(M-1)}^{M-1} T_{p}\left(e^{\mathrm{j} \omega}\right) X\left(\mathrm{j} \Omega-\mathrm{j} \frac{2 \pi p}{M T}\right)
$$

with

$$
T_{p}\left(e^{\mathrm{j} \omega}\right)=\frac{1}{M} \sum_{m=1}^{M} F_{m}\left(e^{\mathrm{j} \omega}\right) H_{m}\left(\mathrm{j} \Omega-\mathrm{j} \frac{2 \pi p}{M T}\right),
$$

where $X(\mathrm{j} \Omega)$ is the Fourier transform of $x(t), \Omega$ the pulsation and $\omega$ the normalized pulsation, $\omega=\Omega T$.

Perfect reconstruction means that the output $y(n)$ is only a scaled, delayed and sampled version of the input. Therefore, perfect reconstruction conditions are [7]:

$$
T_{p}\left(e^{\mathrm{j} \omega}\right)= \begin{cases}c e^{-\mathrm{j} \omega \rho} & , p=0, \rho \in \mathbb{R}_{*}^{+}, c \in \mathbb{R}_{*} \\ 0 & , p \in \mathcal{P} .\end{cases}
$$

where $\rho$ is the overall HFB's delay, $c$ is the scale factor and $\mathcal{P}=\{-(M-1), \ldots-1,1, \ldots, M-1\}$.

Unfortunately, the perfect reconstruction conditions cannot be achieved [7]. So, an approximation should be found taking into account the realization constraints either for analog and digital filters. We define two major performances to characterize the HFB quality reconstruction: the distortion function $R(\omega)$ given in (4) and the aliasing functions $T_{p}\left(e^{j \omega}\right), p \in \mathcal{P}$.

$$
R(\omega)=\frac{T_{0}\left(e^{\mathrm{j} \omega}\right)}{c e^{-\mathrm{j} \omega \rho}}
$$

This distortion function has ideally a magnitude equal to 1 and a phase equal to 0 . Concerning aliasing, each $T_{p}\left(e^{j \omega}\right)$, $p \in \mathcal{P}$, defines an aliasing transfer function which is ideally equal to 0 . Considering aliasing terms as decorrelated noises, we compute the total aliasing terms contribution with:

$$
\operatorname{TotalAliasing}(\omega)=\sqrt{\sum_{p \in \mathcal{P}}\left|T_{p}\left(e^{\mathrm{j} \omega}\right)\right|^{2}}
$$

Also, we define the ratio between the distortion and the total aliasing and note it $D A R(\omega)$ in $\mathrm{dB}$.

$$
\operatorname{DAR}(\omega)=20 \log \left|\frac{R(\omega)}{\text { TotalAliasing }(\omega)}\right|
$$

\section{B. HFB advantages and limitations}

In the context of VersaNum and more generally software radio applications, HFB are interesting because they enlarge the conversion bandwidth for a sampling rate given by available ADCs. Also, they offer versatility in the conversion characteristics such as bandwidth and resolution. Nevertheless, in order to make this solution definitely attractive, the HFB sensitivity to mismatch between analog and digital parts should be overcome.

\section{HFB optimization}

In the literature, some methods synthetize jointly the analog part and the digital part [7] and [4]. These methods require the realization of given analog transfer functions which becomes increasingly difficult as monolithic integration dimensions decrease.

We first studied the influence of analysis filters characteristics. For pure two-order resonators, we made variations on the bandpass, the $Q$-factor and the central frequencies positions. The results show that if we consider the same $Q$-factor for all bands, for a given bandwidth, there is an optimal $Q$-factor value that maximizes the HFB performance while minimizing the quantization noise amplification. As an example, for the bandwidth [400 600] MHz, the optimal $Q$-factor value is 20 . We also studied higher order filters. For example, 6-order Tchebychev filters give a mean $D A R$ of $58 \mathrm{~dB}$ instead of 55 for the two-order resonators. But this improvement is not important enough to compensate the additional analog realization complexity. In VersaNum, the strategy is clearly to maximally relax the constraints upon the analog part realization. So, the analog filters are very simple filters like two-order resonators.

Concerning the synthesis bank optimization, several digital structures and design methods have been investigated. For FIR filters, the method LMSGA [5] is the method that gives the best performances for the HFB. Results show that a slight oversampling of a few percents on the signal Nyquist rate is necessary to obtain a correct HFB performance. Also, the HFB $D A R$ increases linearly with the number of FIR coefficients. The influence of the quantization noise have been studied too. The LMSGA method computes the synthesis filters that minimize the quantization noise amplification. Concerning IIR filters, a previous study [2] provided a synthesis method. A further work shows that for a similar complexity in terms of number of operations, IIR filters give an improvement of $8 \mathrm{~dB}$ compared to FIR filters. However, the recursive part requires to calculate with a much higher resolution. So, in VersaNum, FIR filters are preferred to IIR filters. The synthesis filters are calculated from the knowledge of the analysis filter frequency responses, with the method LMSGA.

\section{Application to VersaNum scenario 1 - WiMax, WiFi case}

A four-channel HFB has been designed for the "multistandard" scenario for a bandwidth of $26.5 \mathrm{MHz}$ with a center frequency of $66 \mathrm{MHz}$. The HFB performs a slightly oversampling of $6 \%$ of the specified $25 \mathrm{MHz}$ band. The highest dynamic range being $82 \mathrm{~dB}$, each elementary ADC should sample at $12.7 \mathrm{MSps}$ with an effective resolution of at least 14 bit. 64-length FIR have been synthetized with the LMSGA method. A mean $D A R$ of $60 \mathrm{~dB}$ is obtained (Figure 2) which is sufficient for the WiFi and WiMax standards (Table I). 


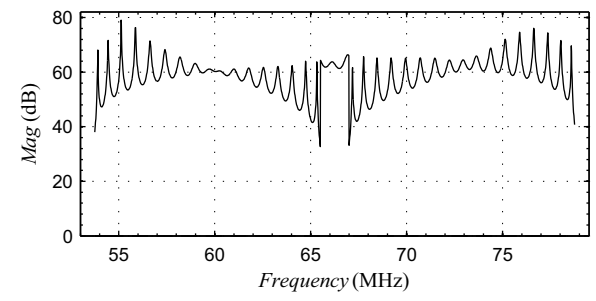

Fig. 2. $D A R(\omega)$ for the wide band reconstruction

\section{E. Application to VersaNum scenario 2 - sensing mode}

An eight-channel HFB has been designed for the "sensing in the TV band" scenario for a bandwidth of $525 \mathrm{MHz}$ with a center frequency of $787 \mathrm{MHz}$. The HFB performs a slightly oversampling of $5 \%$ of the specified $500 \mathrm{MHz}$ band. The highest dynamic range being $82 \mathrm{~dB}$, each elementary ADC should sample at $153 \mathrm{MSps}$ with an effective resolution of at least 14 bit. 128-length FIR have been synthesized with the LMSGA method. A mean $D A R$ of $53 \mathrm{~dB}$ is obtained which is sufficient for the sensing mode according to Table II.

\section{HFB VERSATILITY}

The highest dynamic range required in both scenarios (82 $\mathrm{dB}$ ) is not achieved with HFBs obtained in Section II.D and II.E. These requirements will be achieved by the versatility e.g. "frequency focusing". The first method, presented in [3] consists in a frequency weighting of the LMSGA criteria in order to limit the aliasing much more in an interesting part of the spectrum than in the rest of the spectrum. The second method uses a reconstruction bank like in a subband HFB architecture [1]. In such an architecture (Figure 3), the digital part reconstructs only one subband signal $\hat{s}_{m}(n)$ among $M$. This structure is interesting because it provides a better $D A R$ and a lower quantization noise amplification than a wideband HFB. But it should be noted that it can be applied only if the band of interest is included in a subband.

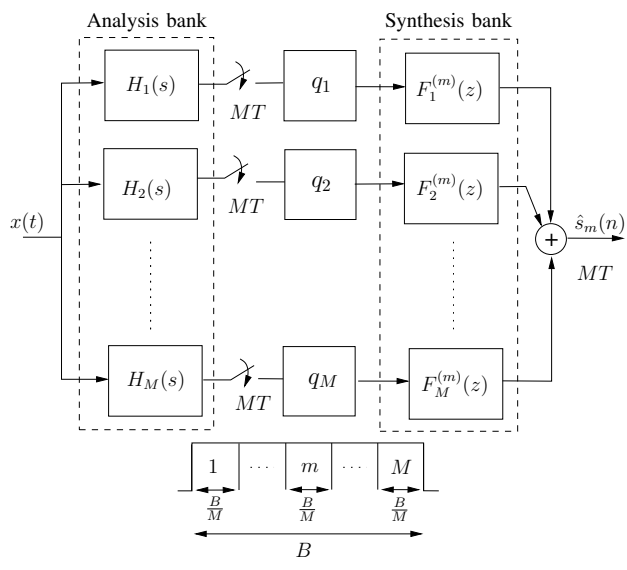

Fig. 3. Subband hybrid filter bank structure

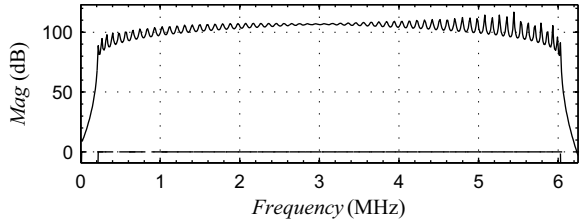

Fig. 4. $D A R(\omega)$ for the reconstruction of the subband [54 59] $\mathrm{MHz}$

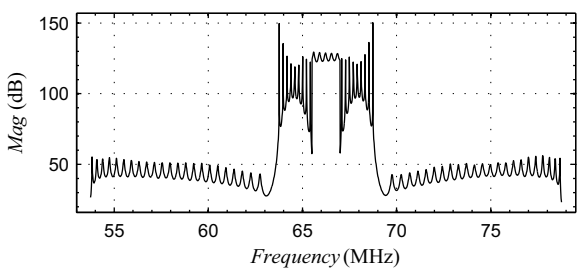

Fig. 5. $D A R(\omega)$ for the reconstruction of the band [63 68] $\mathrm{MHz}$

\section{A. Application to scenario 1 - UMTS, GSM case}

The most restricting case is the UMTS case. Others digital filter banks have been computed starting from the same analog structure as in Section II.D. Two relevant $5 \mathrm{MHz}$ band have been considered. The band [54 59] $\mathrm{MHz}$ being included in a subband, a subband HFB can be used. For the band [63 68] MHz, a subband synthesis cannot be performed so a "frequency focusing" on the wide band signal has been performed. As seen in Table III, results are satisfying for UMTS and consequently GSM. Figure 4 and Figure 5 show the $D A R$ for both bands of interest.

TABLE III

SCENARIO 1 - FREQUENCY FOCUSING IN A 5 MHZ BAND

\begin{tabular}{|c|c|c|c|c|}
\hline $\begin{array}{c}\text { Band of } \\
\text { interest }\end{array}$ & $\begin{array}{c}\text { HFB } \\
\text { type }\end{array}$ & $\begin{array}{c}\text { FIR } \\
\text { length }\end{array}$ & $\begin{array}{c}D A R \text { mean } \\
\text { value }(\mathrm{dB})\end{array}$ & $\begin{array}{c}D A R \text { min } \\
\text { value }(\mathrm{dB})\end{array}$ \\
\hline \hline$[5459 \mathrm{MHz}]$ & subband & 128 & 96 & 80 \\
\hline$[6368 \mathrm{MHz}]$ & wideband & 128 & 118 & 57 \\
\hline
\end{tabular}

\section{B. Application to scenario 2 - Communication mode}

As seen before, the resolution required for the communication mode can't be achieved by the HFB defined in Section II.E. Others digital filter banks have been computed starting from the same analog structure as in Section II.E. Frequency focusing has been applied to improve the resolution. Table IV shows the results for a $10 \mathrm{MHz}$ bandwidth at two different positions. The band [535 545] MHz is included in a subband so that a subband HFB can be used. For the band [650 660] MHz, a subband synthesis cannot be performed so a "frequency focusing" on the wide band signal has been performed. As seen in Table IV, results are satisfying for the communication mode.

\section{HFB CALIBRATION}

The sensitivity of HFB performances to analog errors is certainly the main drawback of HFBs. The chosen strategy is to correct the imperfections of the analog part in the digital 
TABLE IV

SCENARIO 2 - FREQUENCY FOCUSING IN A 10 MHz BAND

\begin{tabular}{|c|c|c|c|c|}
\hline $\begin{array}{c}\text { Band of } \\
\text { interest }\end{array}$ & $\begin{array}{c}\text { HFB } \\
\text { type }\end{array}$ & $\begin{array}{c}\text { FIR } \\
\text { length }\end{array}$ & $\begin{array}{c}D A R \text { mean } \\
\text { value }(\mathrm{dB})\end{array}$ & $\begin{array}{c}D A R \text { min } \\
\text { value }(\mathrm{dB})\end{array}$ \\
\hline \hline$[535545 \mathrm{MHz}]$ & subband & 64 & 133 & 114 \\
\hline$[650660] \mathrm{MHz}$ & wideband & 64 & 128 & 101 \\
\hline
\end{tabular}

part. The developed idea consists in achieving a calibration of the system. At the end of the manufacturing process, a first optimized synthesis filter bank is computed from the measurement of real analog filters. However, the high sensitivity of HFB to mismatches between analog and digital due to temperature variations, component ageing, etc., requires a regular calibration. This calibration should be reasonably implemented in an embedded system.

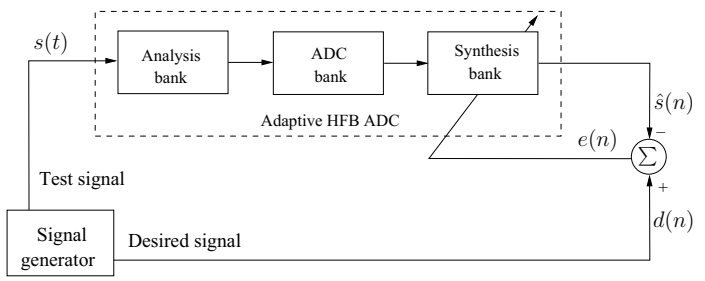

Fig. 6. Principle of the HFB ADC calibration: channel adaptive equalization.

Its principle relies upon an adaptive equalization as shown in Figure 6. A reference signal is entered into the HFB. The synthesis filter coefficients are iteratively adjusted to reconstruct a desired signal. The LMS algorithm has been chosen for its simplicity. This method has been first applied to a subband HFB [6]. Let's consider that the analog center frequencies have been affected by an offset of $1 \%$ of the subband bandwidth. Table IV shows that after the calibration, the performances are satisfying for the considered scenarios. The method is currently applied to a wideband HFB.

TABLE V

SUBBAND HFB CALIBRATION PERFORMANCES

\begin{tabular}{|c|c|c|c|}
\hline \multirow{2}{*}{$\begin{array}{c}\text { Without } \\
\text { Analog imperfections }\end{array}$} & \multicolumn{2}{|c|}{ With analog imperfections } \\
\cline { 3 - 4 } & & $\begin{array}{c}\text { Before } \\
\text { calibration }\end{array}$ & $\begin{array}{c}\text { After } \\
\text { calibration }\end{array}$ \\
\hline$D A R$ mean value & $96 \mathrm{~dB}$ & $53 \mathrm{~dB}$ & $87 \mathrm{~dB}$ \\
\hline
\end{tabular}

\section{HFB PROTOTYPE}

In order to validate the methods in the real world, an HFB prototype has been designed by Thalès. We present here its specification. This prototype is an electronic card realised with discrete components. So it works at a rather low frequency. The goal is to show that, starting from a given ADC technology, the HFB may alternatively convert a signal whose the frequency band is $M$ times larger with a medium resolution and convert a narrow band signal with a high resolution (that is to say that of the elementary ADC). Figure 7 shows the architecture of the prototype and its test environment. The signal to convert is in the band [20-40] MHz. The analog signal is splitted into eight channels through an analog bandpass filter bank (low- $Q$ LC filters). Each channel is sampled with an Analog Device ADC AD9446 (16-bit, 80/100 MSPS ADC) at 5 MSPS rate. A programmable digital circuit Altera Stratix II has been chosen for the digital part and completed by additional SRAM blocks. The digital part of the prototype is configured by a PC. A first development step has validated the analog bandpass filter bank. The final prototype is being manufactured.

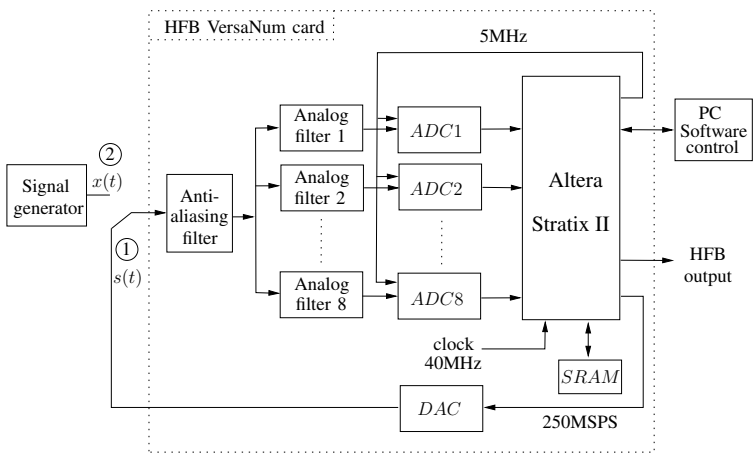

Fig. 7. Principle of HFB ADC calibration: channel adaptive equalization.

\section{CONCLUSION}

The project VersaNum gives the possibility to position HFB $\mathrm{ADC}$ versus future conversion need. The main advantages of HFB compared to a single ADC or even a time-interleaved ADC is its versatility capability. It is possible to convert alternatively wide-band signal with a medium resolution or a narrow band signal with a higher resolution, just by reprogramming the digital part without changing the analog part. The proposed calibration method should be a solution to the sensitivity problem. The prototype will give a practical validation of the developed methods.

\section{REFERENCES}

[1] D. Asemani, J. Oksman, and P. Duhamel, "Subband architecture for hybrid filter bank a/d converters," IEEE Journal of Selected Topics in Signal Processing, vol. 2, no. 2, pp. 191 - 201, April 2008.

[2] C. Lelandais-Perrault, D. Silion, T. Petrescu and D. Poulton "Hybrid Filter Banks A/D Converters using IIR Synthesis Filters", in Proceedings of IEEE Midwest Symposium on Circuits and Systems, August 2005.

[3] C. Lelandais-Perrault, T. Petrescu, D. Poulton, P. Duhamel, J. Oksman, "Wide-band, band pass and versatile Hybrid Filter Bank A/D, conversion for software radio", in IEEE TCAS-I (Transactions Circuits and Systems I), Accepted to publication, 2009.

[4] P. Löwenborg, H. Johansson, and L. Wanhammar, "Two-channel digital and hybrid analog/digital multirate filter banks with very low-complexity analysis or synthesis filters," IEEE Transactions on Circuits and Systems II, vol. 50, no. 7, pp. 355-367, July 2003

[5] T. Petrescu, J. Oksman, and P. Duhamel, "Synthesis of hybrid filter banks by global frequency domain least square solving," in Proceedings of IEEE International Conference on Circuits and Systems, May 2005.

[6] Z. Song, C. Lelandais-Perrault, D. Poulton, and P. Benabes "Adaptive equalization for calibration of subband hybrid filter banks A/D converters," submitted to ECCTD 2009.

[7] S. R. Velazquez, T. Q. Nguyen, and S. R. Broadstone, "Design of hybrid filter banks for analog/digital conversion," IEEE Transactions on Signal Processing, vol. 46, no. 4, pp. 956-967, April 1998. 